\title{
Pseudopapillary tumour of pancreas in a 13 year old girl
}

\author{
Balasundaram Prashanthan, Hasantha Thambawita \\ Teaching Hospital Batticaloa
}

Keywords: Solid pseudopapillary tumour; Frantz's tumour

\section{Introduction}

Solid pseudopapillary tumour of the pancreas is rare. It is a low grade malignant tumour with minimal metastatic potential among the children [1] [2]. The tumour has a good prognosis following complete surgical excision. Here we report a case of a solid pseudopapillary tumour presented as an acute abdomen.

\section{Case report}

A 13 year old previously healthy girl admitted to Teaching Hospital Batticaloa with a history of abdominal pain for 1 week duration, worsened during last 24 hours. It was associated with fever, nausea and vomiting for one day. She had one episode of hematemesis. Urinary and bowel habits were normal. There was no recent weight loss or jaundice. On admission she had a tachycardia $(115 \mathrm{bpm})$ but blood pressure was normal. Abdominal examination revealed an epigastric tender mass. There was guarding but no rigidity. She was clinically pale. Urgent full blood count showed neutrophil leucocytosis $(18,740 / \mathrm{mm} 3)$ with low $\mathrm{Hb}(9.6 \mathrm{~g} / \mathrm{dl})$. Renal, liver profile, urine analysis and coagulation screening were normal. Serum amylase was within normal limits.

ESR was found elevated to $55 \mathrm{~mm} / 1 \mathrm{st} \mathrm{hr}$ as well as the CRP to 46. Urgent ultrasound abdomen showed a $14.3 \mathrm{~cm} * 9.6 \mathrm{~cm} * 10.8 \mathrm{~cm}$ highly vascular heterogenic lesion at epigastric region. She was kept under close monitoring. IV antibiotics were started. Consequent upper gastrointestinal endoscopic study revealed erosive antral gastritis with reflux disease. Computed tomography of the abdomen was not done due to machine failure the parents declined transfer to another institution for this purpose. Exploratary laparotomy revealed highly vascular tumour arising from retro peritoneal area and involving middle body of the pancreas. $12 \mathrm{~cm} * 10 \mathrm{~cm}$ in dimension. Peritoneal survey was negative for any metastatic deposit. The tumour removed with the body of pancreas.

Correspondence: Balasundaram Prashanthan

E-mail: prashanth1983@gmail.com

Received: 09-05-2018 Accepted: 10-06-2018

(iD) http://orcid.org/0000-0003-0911-9940

DOI: http://doi.org/10.4038/sljs.v36i3.8529

The Sri Lanka Journal of Surgery 2018; 36(3): 12-13
The head, neck and tail of the pancreas were preserved considering the child was too young for insulin dependency. Common bile duct, splenic artery portal vein were identified and preserved. The remaining parts of the pancreas were anastomosed to preserve the continuity. Histology revealed capsulated solid pseudopapillary tumour of pancreas with no evidence of capsular or perineural invasion. Areas of necrosis and haemorrhages seen microscopically. Resection margins were negative. Post-operative period and follow up was uneventful. The patient was directed for oncology follow up. But no additional therapy was instituted.

\section{Discussion}

Solid pseudopapillary tumour is referred to as Frantz's tumour after ther person who described it in 1959.

It commonly affects the young and has a female preponderance [1]. It is a low grade malignant neoplasm. Abdominal pain is a common initial presentation but some present with asymptomatic abdominal mass [2].This patient presented with abdominal pain and the abdominal mass elicited by the clinical examination. Elevated amylase levels were observed in some cases, but this patient's serum amylase was within normal limit. In contrast to the adults, children with solid pseudopapillary tumour have a low distant metastatic potential which corresponds with our case as well [3] [4].

The tumour commonly involves pancreatic tail followed by the head, and the involvement of the body is rare. But in this case the tumour was localized to the body, sparing head and tail [4]. Computed Tomography (CT) of abdomen was done preoperatively in most of the literature[3][4]. Surgical resection without preoperative $\mathrm{CT}$ was challenging for us. Therefore doing preoperative imaging will be a benefit for surgeon to analyse.

But our case due to the above mentioned reasons, deviated from the routine norm. Even-though in the early years pancreatoduodenectomy was performed, newer studies recommend enucleation of the tumour or partial pancreatectomy [4]. Also literature recommends complete surgical resection of the tumour alone is the best treatment [4]. In this case, we managed the patient alone with complete surgical resection of tumour as with the literature and the postoperative follow up was uneventful. 


\section{Conclusions}

Solid Pseudopapillary tumour of pancreas is a low grade malignant disease of with minimal metastatic potential. Most of the time surgical enucleation alone will be sufficient.

All authors disclose no conflict of interest. The study was conducted in accordance with the ethical standards of the relevant institutional or national ethics committee and the Helsinki Declaration of 1975, as revised in 2000 .

\section{References}

1. Siquini W, Marmorale C, Guercioni G, Stortoni P, Bearzi I, Fianchini A et al. [Solid pseudopapillary tumor of the pancreas. A report of 3 cases and a review of the literature]. [Internet]. Europepmc.org. 2018 [cited 10 March 2018]. Available from: http://europepmc.org/abstract/med/16734174

2. Ayadi L, Ellouze S, Khabir A, Daoud E, Bahri I, Trigui D et al. [Frantz's tumor: anatomoclinical study of six Tunisian cases] [Internet]. Europepmc.org. 2018 [cited 10 March 2018]. Available from: http://europepmc.org/abstract/med/19202714

3. Escobar MA, Bond BJ, Schopp J. Solid pseudopapillary tumour (Frantz's tumour) of the pancreas in childhood. BMJ Case Reports. 2014;2014:bcr2013200889. doi:10.1136/bcr-2013200889

4. Jurić I, Pogorelić Z, Stepan JG, Kuzmić IP. Extremely rare presentation of Frantz's tumour: synchronous localisation in the pancreatic head and tail. Scott Med J [Internet]. 2014 [cited 17Mar.2018];59 (3 ):e8-e12. Available from: http://dx.doi.org/10.1177/0036933014543222

\section{Learning Points}

- Solid Pseudopapillary tumour commonly present with abdominal pain but asymptomatic cases also reported.

- Common sites of origin are Pancreatic tail and head in the descending order.

- It has a low metastatic potential in children.

- Preoperative imaging of the tumour would be a fringe benefit for surgery.

- Most of the time surgical enucleation alone will be sufficient. 